Nonlinear Processes in Geophysics, 12, 661-670, 2005

SRef-ID: $1607-7946 / \mathrm{npg} / 2005-12-661$

European Geosciences Union

(C) 2005 Author(s). This work is licensed

under a Creative Commons License.

\title{
Non-linear complex principal component analysis of nearshore bathymetry
}

\author{
Sanjay S. P. Rattan ${ }^{1, *}$, B. G. Ruessink ${ }^{2}$, and W. W. Hsieh ${ }^{1}$ \\ ${ }^{1}$ Dept. of Earth and Ocean Sciences, University of British Columbia, Vancouver, B.C., Canada, V6T 1Z4 \\ ${ }^{2}$ Dept. of Physical Geography, Faculty of Geosciences, Institute for Marine and Atmospheric Research, Utrecht University, \\ P.O. Box 80.115, 3508 TC Utrecht, Netherlands \\ * present address: Department of Earth and Atmospheric Sciences, University of Alberta, Canada
}

Received: 24 January 2005 - Revised: 7 March 2005 - Accepted: 13 May 2005 - Published: 28 June 2005

\begin{abstract}
Complex principal component analysis (CPCA) is a useful linear method for dimensionality reduction of data sets characterized by propagating patterns, where the CPCA modes are linear functions of the complex principal component (CPC), consisting of an amplitude and a phase. The use of non-linear methods, such as the neural-network based circular non-linear principal component analysis (NLPCA.cir) and the recently developed non-linear complex principal component analysis (NLCPCA), may provide a more accurate description of data in case the lower-dimensional structure is non-linear. NLPCA.cir extracts non-linear phase information without amplitude variability, while NLCPCA is capable of extracting both. NLCPCA can thus be viewed as a non-linear generalization of CPCA. In this article, NLCPCA is applied to bathymetry data from the sandy barred beaches at Egmond aan Zee (Netherlands), the Hasaki coast (Japan) and Duck (North Carolina, USA) to examine how effective this new method is in comparison to CPCA and NLPCA.cir in representing propagating phenomena. At Duck, the underlying low-dimensional data structure is found to have linear phase and amplitude variability only and, accordingly, CPCA performs as well as NLCPCA. At Egmond, the reduced data structure contains non-linear spatial patterns (asymmetric bar/trough shapes) without much temporal amplitude variability and, consequently, is about equally well modelled by NLCPCA and NLPCA.cir. Finally, at Hasaki, the data structure displays not only non-linear spatial variability but also considerably temporal amplitude variability, and NLCPCA outperforms both CPCA and NLPCA.cir. Because it is difficult to know the structure of data in advance as to which one of the three models should be used, the generalized NLCPCA model can be used in each situation.
\end{abstract}

Correspondence to: B. G. Ruessink

(g.ruessink@geo.uu.nl)

\section{Introduction}

Like data sets in many other branches of science, nearshore bathymetric data sets are highly dimensional, being composed of numerous observations in time at a considerable number of spatial positions. Along sandy beaches, these highly dimensional data sets often show the evolution of submarine ridges of sand (sandbars) and their intermediate lows (sandbar troughs). Principal component analysis (PCA) (Von Storch and Zwiers, 1999; Jolliffe, 2002) is an often applied technique in nearshore batymetric studies to reduce the data dimensionality with the purpose of gaining more insight into the dynamics of these bars (e.g., Wijnberg and Terwindt, 1995; Kuriyama, 2002). However, PCA suffers from a number of drawbacks that cause this technique to not always be the most useful technique for dimensionality reduction in nearshore (as well as in other (non-) geophysical) studies.

In sandbar studies, these drawbacks are related to the propagating character of the sandbars and to their often asymmetric (non-linear) spatial shape. This propagating character expresses itself as bar generation near the shoreline, followed by offshore migration until a bar finally dies away at the outer edge of the nearshore (Ruessink and Kroon, 1994; Shand and Bailey, 1999; Plant et al., 1999; Kuriyama, 2002). Because PCA is limited to the detection of standing spatial patterns, PCA scatters the propagating sandbars in two or more PCA modes, complicating their physical interpretation. While complex PCA (Horel, 1984) (where a mode is of the functional form $\mathbf{L}\left(a e^{i \theta}\right)$, with $\mathbf{L}$ linear, and the complex principal component having an amplitude $a$ and a phase $\theta$ ) may overcome this problem (e.g., Ruessink et al., 2003), the linearity assumption in CPCA imposes symmetric sandbar and trough shapes, contrasting with often observed asymmetric bar/trough profiles. Ruessink et al. (2004), for instance, noted that bars along parts of the Dutch coast can be narrower and higher than the relatively wide and shallow 
troughs. Linear methods of dimensionality reduction now lead to modes that are uncorrelated but not independent and, as a consequence, cannot be interpreted separately.

The advent of multi-layer perceptron neural networks (Gardner and Dorling, 1998; Hsieh and Tang, 1998), henceforth denoted NNs, resulted in the non-linear generalization of PCA (Kramer, 1991; Malthouse, 1998; Hsieh, 2001) and CPCA (Rattan and Hsieh, 2004, 2005). Of particular relevance for the description of propagating features are the NN-based techniques circular non-linear PCA (NLPCA.cir) (Kirby and Miranda, 1996; Hsieh, 2001) and the recently developed non-linear CPCA (NLCPCA) (Rattan and Hsieh, 2004, 2005). NLPCA.cir and NLCPCA differ in the form of non-linearity they can describe. An NLPCA.cir solution contains non-linear phase information (as an NLPCA.cir mode is of the functional form $\mathbf{N}(\theta)$, with $\mathbf{N}$ non-linear) but cannot describe temporal variability in the magnitude of the extracted features. Thus, while NLPCA.cir can be successful in condensing the asymmetric shape of sandbars and troughs in one mode (Ruessink et al., 2004), any temporal variability in the magnitude of this shape remains unresolved and may seriously impair the description of the lower-dimensional data structure when this variability is considerable (cf. Hamilton and Hsieh, 2002). NLCPCA (with a non-linear functional form of $\mathbf{N}\left(a e^{i \theta}\right)$ ) overcomes this deficiency in NLPCA.cir and captures both the phase and amplitude variability nonlinearly. For a review of non-linear PCA and for oceanographic and climatological applications, see Hsieh (2004).

In this study, the NLCPCA method is applied to the bathymetry data recently used in Ruessink et al. (2004) to investigate how effective this method is in comparison to the CPCA and the NLPCA.cir methods in representing propagating phenomena. As the first application of the NLCPCA method to coastal morphodynamics, this paper illustrates the potential advantage of NLCPCA over CPCA and NLPCA.cir in analyzing geophysical (or non-geophysical) datasets.

\section{Data}

The bathymetric data used in this paper were collected at the sandy barred beaches of Egmond aan Zee (The Netherlands), the Hasaki Coast (Japan) and Duck (North Carolina, USA). Details on data acquisition and processing are given by Ruessink et al. (2004) and are only briefly reiterated here. For Egmond, 122 observations at 151 cross-shore positions were collected between 1964 and 2001 at weekly to annual intervals using vertical aerial photogrammetry and echosounding (e.g., Wijnberg and Terwindt, 1995). Data at Hasaki (Kuriyama, 2002), comprising 1414 observations at 85 cross-shore positions, were made from a $427 \mathrm{~m}$-long pier facing the Pacific Ocean on a daily basis between 1987 and 1991, while Duck data (423 observations, 121 cross-shore positions) were collected at the U.S. Army Corps of Engineers Field Research Facility on a fortnightly to monthly basis (1981-2002) using an amphibuous vehicle (e.g., Birke- meier and Mason, 1984; Plant et al., 1999). At all three sites the spacing between consecutive cross-shore positions is $5 \mathrm{~m}$.

From all available time series (that is, time series of depth at each cross-shore location $x$ ) the time-averaged depth value was removed, resulting in perturbation datasets, in which bars (troughs) are associated with positive (negative) perturbations. Time-space diagrams of the perturbation data sets are provided in the upper row of Fig. 1. Warm colours (red) correspond to positive perturbations (bars), cold colours (blue) reflect negative perturbations (troughs). All 3 sites are characterized by sandbars that have multiannual lifetimes and behave in an interannual quasi-periodic offshore directed manner, as described in Ruessink and Kroon (1994). A bar cycle comprises bar birth in the inner nearshore, followed by up to several years of net offshore migration and final disappearance in the outer nearshore zone. The disappearance of the outer bar triggers the onset of net offshore migration of the next most seaward located bar, and the generation of a new bar near the shoreline, as is clearly demonstrated for Egmond around, for instance, 1970 and 1985. The cycle duration, i.e. the time period between successive bar disappearance, varies from site to site (12 years in Egmond, 1 year in Hasaki and 3.5 years in Duck) (Ruessink et al., 2003). Discussions on the relationship between the cycle return period and bulk statistics of external forcings (wave, tide, wind, sediment, and bed profile characteristics) can be found in Shand et al. (1999); Kuriyama (2002) and Ruessink et al. (2003).

As can be seen in the time-space diagrams, bars typically reach their maximum amplitude (dark red colour) in the mid nearshore, with smaller values further near the shoreline and further offshore. The distance between consecutive bar crests (in the cross-shore) increase in the offshore direction from $\approx 100 \mathrm{~m}$ near the shore to several hundreds of meters further offshore, see Ruessink et al. (2003) for further discussion. It is striking to see that the offshore progression of the bars at Egmond is relatively smooth in time, whereas that at Duck and, to a smaller extent, at Hasaki is characterized by frequent standstills or short-term (up to several seasons) onshore-directed progression. During such onshore migration phases, the bars may disappear almost entirely (e.g. 1982-1983 at Duck), only to increase again during the next winter season when the offshore bar migration resumes. On the whole, it thus appears that the temporal variability in bar amplitude at Duck and Hasaki exceeds that at Egmond.

Environmental characteristics at the three sites are listed in Table 1 and include the mean slope $\beta$ of the barred part of the profile (Ruessink et al., 2003), the median grain size $D_{50}$, the "storm" breaker height $H_{b}$ and peak period $T_{p}$, the low-tide level $\eta_{\mathrm{LT}}$ with respect to mean sea level and the tide range $\Delta \eta=\eta_{\mathrm{HT}}-\eta_{\mathrm{LT}}$, where $\eta_{\mathrm{HT}}$ is the high-tide level. The $D_{50}$ is often not constant in the cross-shore (e.g., Katoh and Yanagishima, 1995) and the values in Table 1 represent midnearshore values. The storm $H_{b}$ and $T_{p}$ were quantified as the 0.99-quantile of time series of $H_{b}$ and $T_{p}$, where the $H_{b}$ time series were computed from long-term, hourly to twohourly sampled offshore wave records collected at each site 

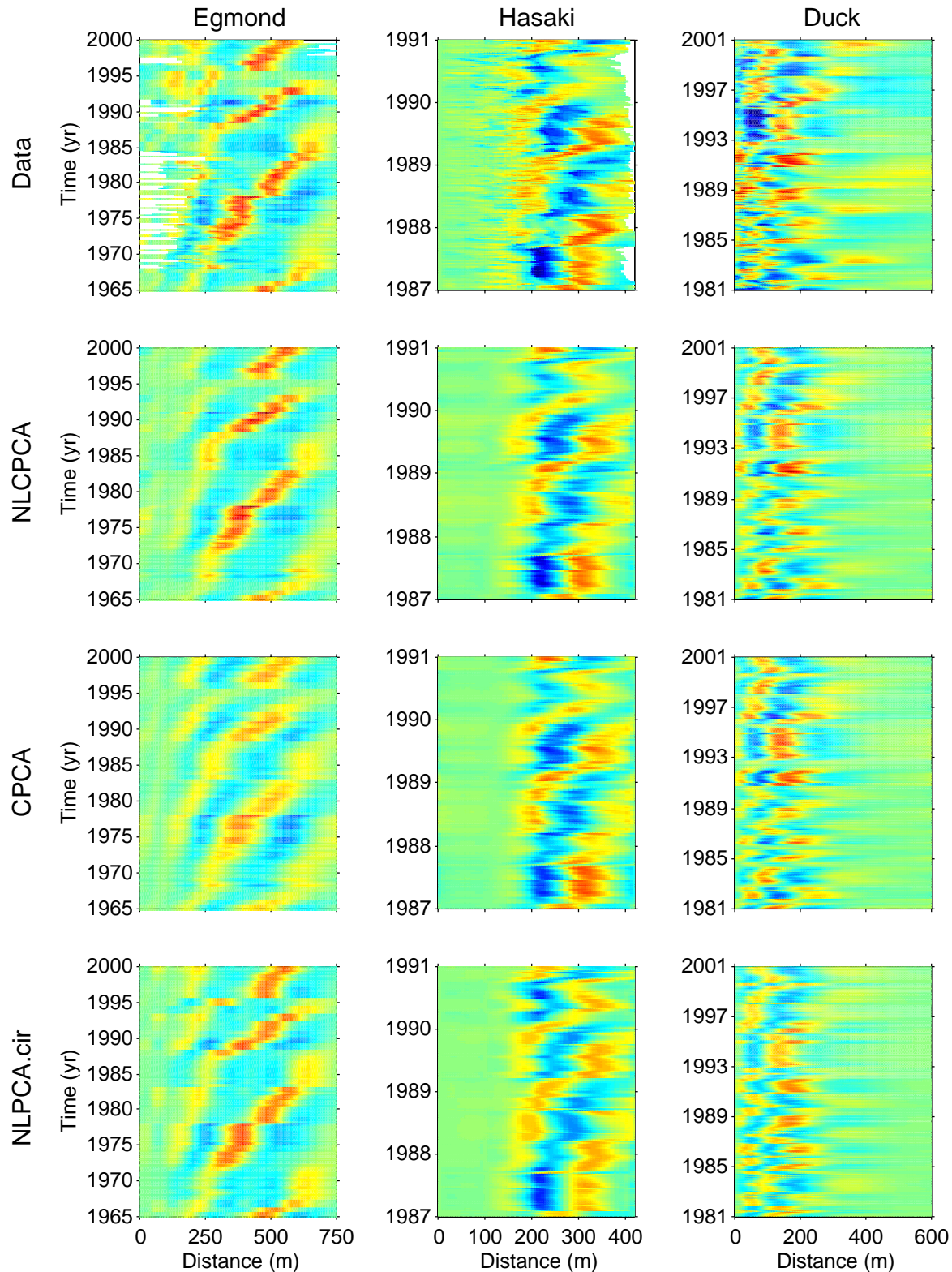

Fig. 1. Observed (top row), NLCPCA mode 1 (second row), CPCA mode 1 (third row) and NLPCA.cir mode 1 (fourth row) perturbations at (first column) Egmond, (second column) Hasaki and (third column Duck). Colours range from -3 (blue) to 3 m (red) at Egmond and Hasaki, and from -1.5 to $1.5 \mathrm{~m}$ at Duck. These different ranges reflect the differences in magnitude of the bars at the three sites. The white colour in the observations at Egmond and Hasaki represent missing values.

using linear wave theory

$H_{b}=\left(\frac{\gamma}{g}\right)^{1 / 5}\left[H_{0}^{2} c_{g 0} \cos \theta_{0}\right]^{2 / 5}$

where $H_{0}, c_{g 0}$, and $\theta_{0}$ are the offshore root-mean-square wave height, group velocity and angle of incidence, respectively. The breaker parameter $\gamma$ was set to 0.4 (Thornton and Guza, 1982). At Hasaki $\theta_{0}=0$ was assumed because wave direction estimates were not available. Values of $\eta_{\mathrm{LT}}$ and $\eta_{\mathrm{HT}}$ were computed as the 0.05 and 0.95 -quantile of long-term water level records obtained at each site, respectively.
Table 1. Environmental characteristics

\begin{tabular}{lcccccc}
\hline Region & $\begin{array}{c}D_{50}, \\
\mu \mathrm{m}\end{array}$ & $\beta$ & $\begin{array}{c}H_{b}, \\
\mathrm{~m}\end{array}$ & $\begin{array}{c}T_{p}, \\
\mathrm{~s}\end{array}$ & $\begin{array}{c}\eta_{\mathrm{LT}}, \\
\mathrm{m}\end{array}$ & $\begin{array}{c}\Delta \eta, \\
\mathrm{m}\end{array}$ \\
\hline Egmond & 170 & $1: 170$ & 2.58 & 9.0 & -0.85 & 1.9 \\
Hasaki & 175 & $1: 75$ & 2.72 & 13.4 & -0.54 & 1.1 \\
Duck & 180 & $1: 80$ & 2.47 & 15.4 & -0.54 & 1.4 \\
\hline
\end{tabular}




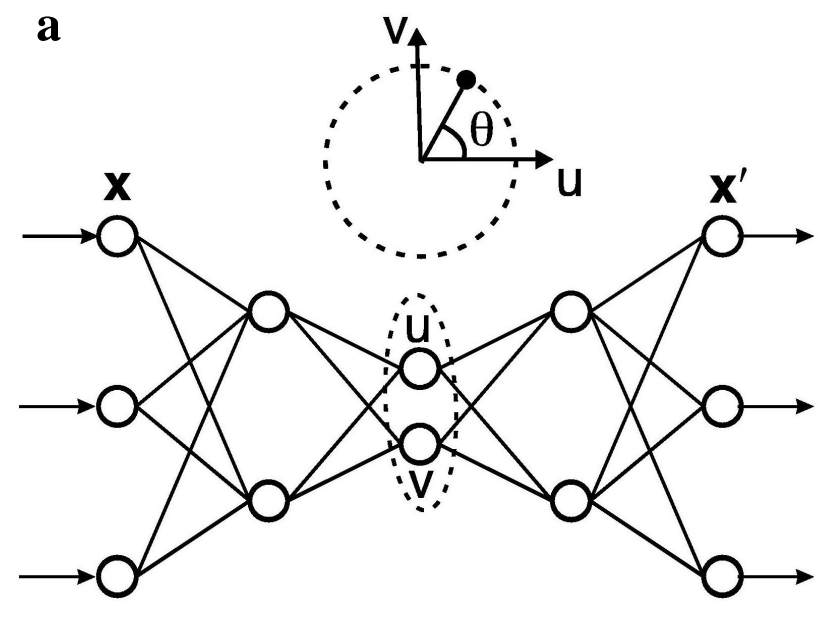

b

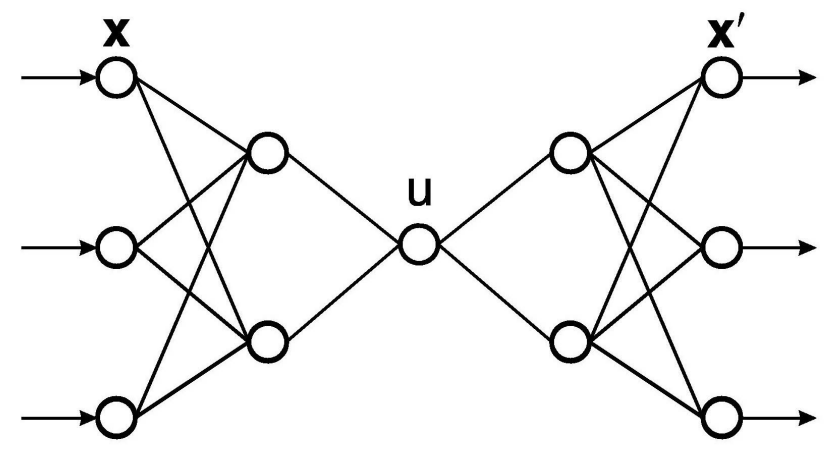

Fig. 2. Schematic diagrams illustrating the $\mathrm{NN}$ model for performing (a) circular non-linear principal component analysis (NLPCA.cir) and (b) complex non-linear principal component analysis (NLCPCA)

\section{Method}

\subsection{CPCA}

CPCA is the complex generalization of PCA (Horel, 1984; Von Storch and Zwiers, 1999). In PCA, a data matrix X with dimension $m \times n$ is decomposed into matrices of principal components and eigenvectors. If we take $n$ to be the number of time points and $m$ the number of spatial points, with zero mean in $n$, then a PCA of $\mathbf{X}$ seeks a solution that contains $r(r \leq m, n)$ linearly independent vectors or eigenvectors in the columns of $\mathbf{Q}(m \times r)$ such that (Strang, 1988)

$\mathbf{X}=\mathbf{Q A}$,

where the rows of $\mathbf{A}(r \times n)$ contain the $r$ principal component (PC) time series. This decomposition is usually performed using the singular value decomposition (SVD) technique (Strang, 1988). To perform CPCA, the data matrix $\mathbf{X}$ is first Hilbert transformed (Horel, 1984; Von Storch and Zwiers, 1999) to yield a matrix $\mathbf{H}[\mathbf{X}]$. The resultant complex matrix $\mathbf{Z}=\mathbf{X}+i \mathbf{H}[\mathbf{X}]$ is then analyzed with CPCA using the same SVD technique used for PCA except that the variables are now complex numbers. Each complex principal component (CPC) has both an amplitude $a$ and a phase $\theta$.

\subsection{NLPCA.cir}

NLPCA.cir uses a standard auto-associative feedforward NN (i.e. multi-layer perceptron), with 3 "hidden" layers of variables or "neurons" (denoted by circles in Fig. 2a) sandwiched between the input layer $\mathbf{x}$ on the left and the output layer $\mathbf{x}^{\prime}$ on the right. In short, the five-layer network can be considered as the combination of two three-layer feedforward NNs, where the third layer of the first sub-network is identical to the first layer of the second sub-network. The first subnetwork maps the input on to NLPC space, and the second sub-network performs the inverse mapping back into original space. The middle layer in the combined network has fewer neurons than the other layers and is therefore known as the bottleneck layer. This, combined with the fact that the $\mathrm{NN}$ is trained to reproduce its inputs, forces the third layer to represent the information in the inputs before it can be decoded by the fourth layer. Thus, a total of 4 layers of transfer functions are needed to map from the inputs to the outputs. Both the second (encoding) and fourth (decoding) layer contain $q$ neurons. A neuron $v_{i}$ at the $i$ th layer receives its value from the neurons $\mathbf{v}_{i-1}$ in the preceding layer, i.e. $v_{i}=f_{i}\left(\mathbf{w}_{i} \cdot \mathbf{v}_{i-1}+b\right)$, where $\mathbf{w}_{i}$ is a vector of weight parameters and $b$ a bias parameter, and the transfer functions $f_{1}$ and $f_{3}$ are the hyperbolic tangent functions, while $f_{2}$ and $f_{4}$ are simply the identity functions. Effectively, a nonlinear function $u=F(\mathbf{x})$ maps from the higher dimension input space to the lower dimension bottleneck space, followed by an inverse transform $\mathbf{x}^{\prime}=\mathbf{G}(u)$ mapping from the bottleneck space back to the original space, as represented by the outputs. To make the outputs as close to the inputs as possible, the cost function $J=\left\langle\left\|\mathbf{x}-\mathbf{x}^{\prime}\right\|^{2}\right\rangle$ (i.e. the mean square error, MSE) is minimized (where $\langle\cdots\rangle$ denotes a sample or time mean). Through the optimization, the values of the weight and bias parameters are solved.

To enable the description of propagating features the bottleneck layer contains a circular neuron (Kirby and Miranda, 1996), comprising two neurons $u$ and $v$ constrained to lie on a unit circle in the $u-v$ plane (Fig. 2a). Thus, NLPCA.cir has only one free angular variable, the angular NLPC $\theta=\arctan (v / u)$. Because of the circular neuron and the non-linear transfer functions $f_{1}$ and $f_{3}$, NLPCA.cir can describe non-linear phase variability but lacks the ability to model temporal amplitude variability. Thus, NLPCA.cir extracts a mode that is in general a nonlinear function of $\theta$.

The presence of multiple minima in the cost function means that an optimization run could end at a local minimum, thereby necessitating multiple runs to solve the optimal (weight and bias) parameters. Here, an ensemble of 25 runs with random initial parameters was used, where for each ensemble member $20 \%$ of the data was randomly selected as test data and withheld from the training of the $\mathrm{NN}$. Runs where the mean square error was larger for the test data 
set than for the training data set were rejected to avoid overfit solutions (Bishop, 1995). The chosen 20\% is a compromise between training and testing. If a substantially larger percentage is used, the NN may no longer capture all relevant features, causing it to have poor generalization properties. On the other hand, a lower percentage may cause the test data to have different statistical properties than the entire data and, accordingly, it loses its relevance in determining whether a specific solution is overfit. To avoid the risk of overfitting, a regularization or weight penalty term was added to the cost function $J$. This term is basically the sum of the square of the model weight parameters multiplied by a user specified "weight penalty" parameter $P$. When $P$ is increased, the model is forced to find solutions with smaller weights, corresponding to less non-linear solutions which are less likely to overfit the data. In the present paper, NNs with the number of encoding neurons $q=1$ to 10 were run, each with $P$ in multiples of 0.001 and 0.01 , and the $\mathrm{NN}$ with the smallest mean square error was then chosen as the best solution.

Because the dimensionality of each perturbation data set is too large for direct use as input into an NLPCA.cir model, PCA was performed first on the input data. The number of parameters to be estimated for even the most simple network architecture $(q=2)$ exceeds the number of temporal observations, leaving an ill-conditioned problem. At all three sites the five leading PCs, representing $85-90 \%$ of the total variance, were used as input into the NLPCA.cir model. It is possible that some signal may be lost in the PCA compression; however, the results of Ruessink et al. (2004) suggest that this is highly unlikely. Measurement errors in the depth values, estimated crudely at about $0.2 \mathrm{~m}$ (Ruessink et al., 2004), are likely to constitute to the non-selected higher-numbered PCA modes and, accordingly, do not influence the NLPCA.cir results presented below. Finally, we note that the choice of five PCA modes is less stringent than in earlier PCA investigations of bathymetric data, which typically used only two PCA mode to describe bar behaviour (e.g., Wijnberg and Terwindt, 1995; Kuriyama, 2002).

\subsection{NLCPCA}

The NLCPCA model has the same NN structure as NLPCA.cir with the exceptions that the bottleneck layer contains an ordinary neuron $u$ (Fig. 2b) and that all NLCPCA parameters and neurons are complex numbers. The single complex-valued bottleneck neuron (the nonlinear complex PC, NLCPC) is unconstrained with both a temporal amplitude (magnitude) and a spatial phase. Consequently, NLCPCA captures both phase and amplitude information nonlinearly. The nonlinear complex transfer function used in the NLCPCA model is the hyperbolic tangent $(\tanh (z))$, with certain constraints on $z$ as described in Rattan and Hsieh (2004, 2005). The NN parameters are randomly initialized with magnitude $\leq 0.1$. Similar to the NLPCA.cir case, the NLCPCA model was run in an ensemble mode because of local minima in the cost function, and contained a weight penalty term scaled with $P$ to avoid the risk of overfit so-
Table 2. Explained variance

\begin{tabular}{lccc}
\hline & Egmond & Hasaki & Duck \\
\hline NLCPCA mode 1 & 81.4 & 78.8 & 60.7 \\
CPCA mode 1 & 66.4 & 71.7 & 59.1 \\
NLPCA.cir mode 1 & 80.8 & 73.1 & 52.2 \\
\hline
\end{tabular}

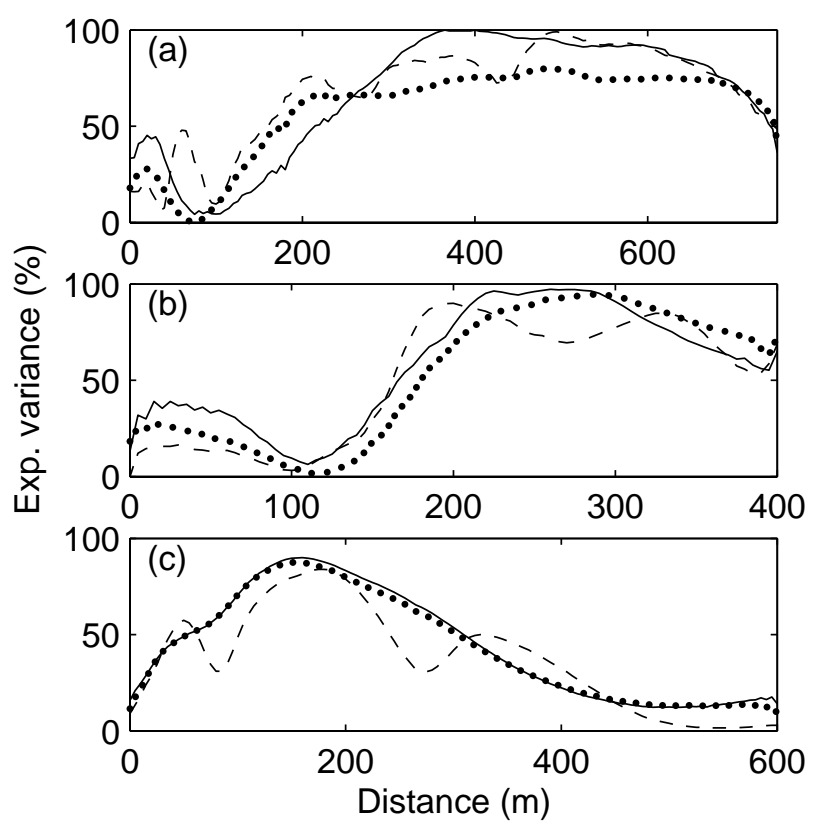

Fig. 3. Explained variance of the perturbation data set by the first (solid line) NLCPCA, (dashed line) NLPCA.cir and (dotted line) CPCA mode versus cross-shore distance at (a) Egmond, (b) Hasaki and (c) Duck

lutions. Rather than the first five leading PCs, the first five leading CPCs were used as input into each NLCPCA model.

\section{Results}

\subsection{Egmond}

The best NLCPCA solution of Egmond was found with $q=7$ and $P=0.002$. The NLCPCA mode 1 explains $81.4 \%$ of the variance in the Egmond perturbation data set, about the same as explained by the leading NLPCA.cir $(q=4$ and $P=0.02)$ mode and about $15 \%$ more than the first linear CPCA mode (Table 2). Both non-linear methods thus lead to a more complete characterization of the lower-dimensional data structure than possible with CPCA. When the fraction of explained variance is viewed as a function of cross-shore distance (Fig. 3a), we see that NLCPCA mode 1 explains more than $65 \%$ of the variance in the zone where bars are most pronounced $(x \approx 250-700 \mathrm{~m})$, with a maximum value of $99 \%$. In comparison to the fraction of variance explained 

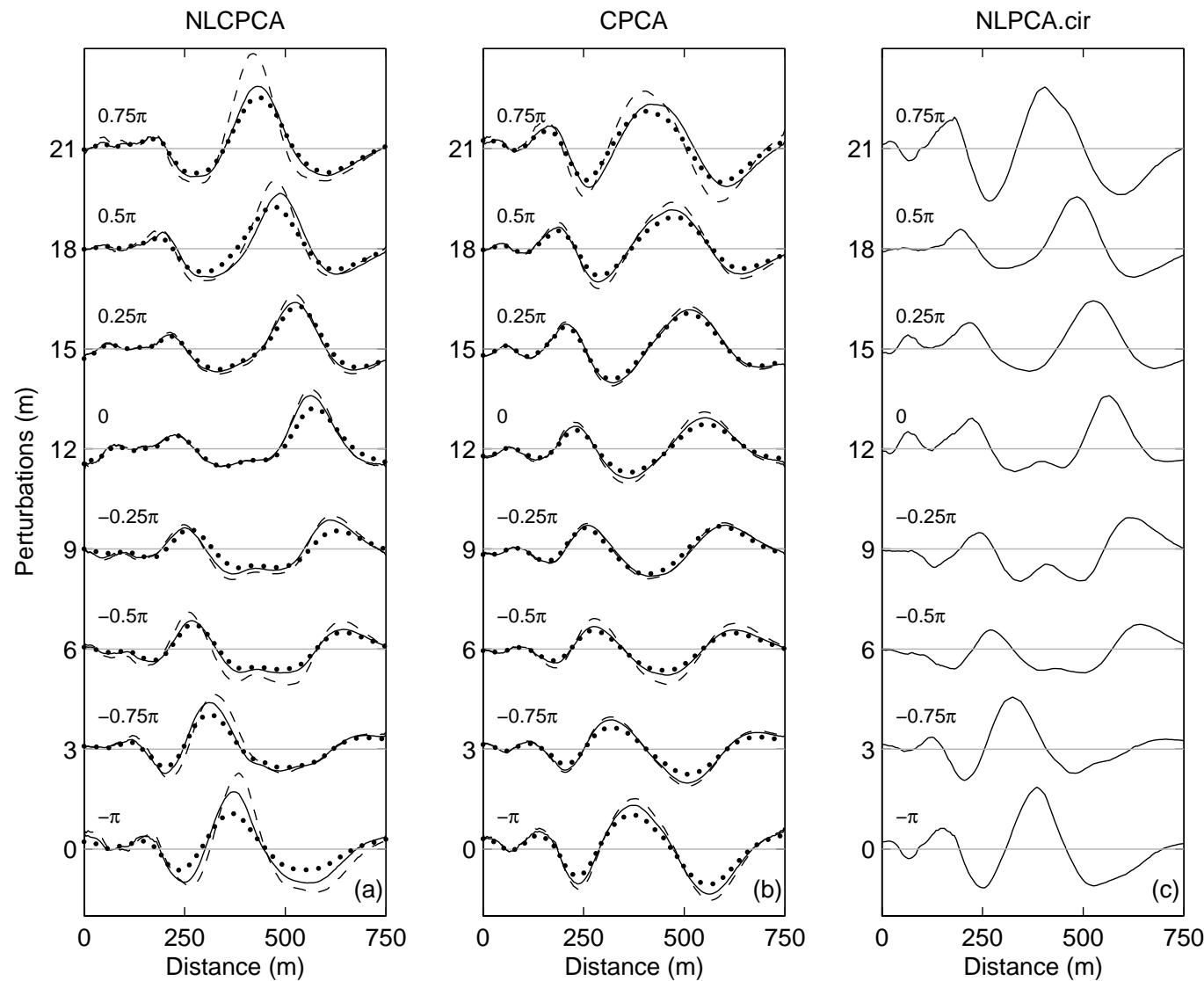

Fig. 4. Sequence of perturbation profiles at Egmond in $\pi / 4$-wide $\theta$ classes centered around $\theta=-\pi$ to $\theta=0.75 \pi$ based on (a) NLCPCA mode 1, (b) CPCA mode 1 and (c) NLPCA.cir mode 1. The three lines in each phase class in (a) and (b) represent the mean perturbation profile for the (dotted line) lower $1 / 3$, (solid line) middle $1 / 3$ and (dashed line) upper $1 / 3$ of the temporal amplitudes in that class. The results for each phase class have been vertically shifted for better visualization.

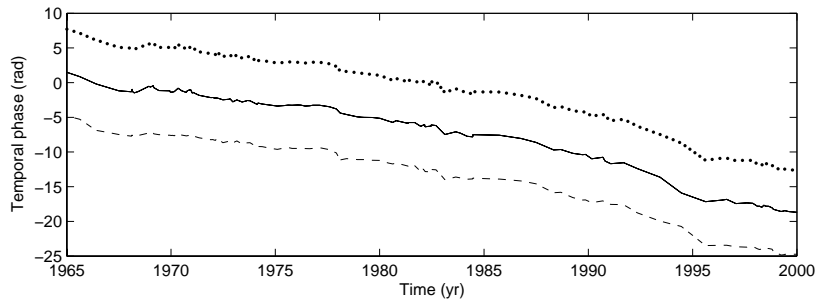

Fig. 5. Temporal phase $\theta$ of (solid line) NLCPCA mode 1, (dashed line) NLPCA.cir mode 1 and (dotted line) CPCA mode 1 versus time at Egmond. The phases are offset by $2 \pi$ for clarity.

by NLPCA.cir and CPCA mode 1, the NLCPCA mode 1 performs less well in the zone where bars are generated $(x \approx 100-250 \mathrm{~m})$.

The larger amount of variance explained by NLCPCA and NLPCA.cir mode 1 relative to the first linear (CPCA) mode implies that the lower-dimensional structure of the Egmond data is non-linear. To investigate the form of this nonlinearity, the perturbations reconstructed with each method (Fig. 1) were divided in $8 \theta$ classes, each $\pi / 4$ in width, where $\theta$ is the temporal phase computed by each method. In each class, the perturbations were sorted by temporal amplitudes and computed into the mean spatial perturbations for the lower $33.3 \%$, middle $33.3 \%$ and upper $33.3 \%$ temporal amplitudes. In this way, we can see how the shape of the perturbations changes with temporal phase and, within each phase class, with temporal amplitude. Because temporal amplitudes for the NLPCA.cir method are 1 by definition, no amplitude sorting can be performed for this method and, accordingly, all perturbations in each phase class were processed into a single class-averaged perturbation profile.

As can be seen in Fig. 4, the bars produced by the two non-linear methods are higher and narrower than those reconstructed by the linear method, whereas the troughs are typically shallower and broader, consistent with the observations. Note, for instance, that in Fig. 1 the Egmond bars modeled by NLCPCA and NLPCA.cir mode 1 have reddish colours, as do the observed bars. The CPCA mode 1 reconstructed bars are generally lower, indicated by the yellowish colours, underestimating the observed bar heights. Thus, there is clear non-linearity in the spatial phase variability in the Egmond sandbar data set. Non-linearity associated with temporal amplitude changes within a phase class is also ob- 

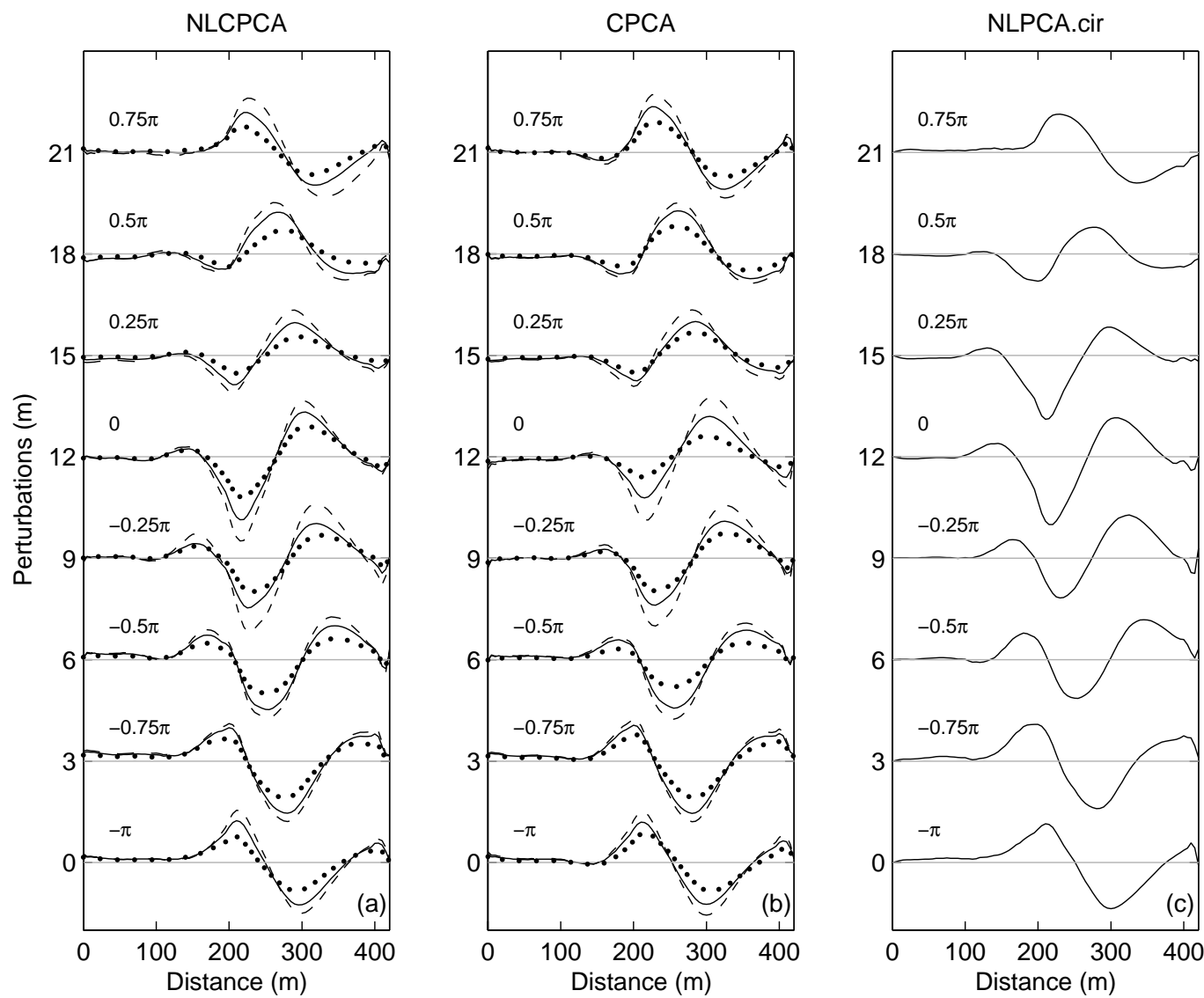

Fig. 6. Sequence of perturbation profiles at Hasaki in $\pi / 4$-wide $\theta$ classes centered around $\theta=-\pi$ to $\theta=0.75 \pi$ based on (a) NLCPCA mode 1, (b) CPCA mode 1 and (c) NLPCA.cir mode 1. The three lines in each phase class in (a) and (b) represent the mean perturbation profile for the (dotted line) lower 1/3, (solid line) middle $1 / 3$ and (dashed line) upper $1 / 3$ of the temporal amplitudes in that class. The results for each phase class have been vertically shifted for better visualization.

vious in Fig. 4. Especially when the bar is located in the midnearshore (e.g. at $x \approx 400 \mathrm{~m}$ in the $0.75 \pi$ phase class), the bar shape in the upper $33.3 \%$ amplitude group departs far more from the bar in the other 2 amplitude groups (Fig. 4a) than in the corresponding CPCA situation (Fig. 4b). It is odd that the CPCA curves for phase class $0.75 \pi$ also change shape for different amplitudes (Fig. 4b), as we expect the CPCA curve to change shape only when the phase is changed. However, because the number of observations at Egmond is rather low (on average, $122 / 8 \approx 15$ per phase class) the average phase of the observations with large amplitudes turned out be slightly different from the average phase for data points with small amplitude. This implies that for Egmond we may never have isolated the amplitude effect from the phase effect fully. On the whole, the about equal amount of explained variance by NLCPCA and NLPCA.cir suggests that the non-linearity in the Egmond data is primarily caused by spatial phase variability, as NLPCA.cir does not model temporal amplitude variability.

The time series of temporal phase extracted by each method are virtually identical (Fig. 5, linear correlation coefficient $>0.99$ ). The general $\theta$ decrease in time implies that the patterns in Fig. 4 evolve from top to bottom and thus describe offshore bar propagation. The slope of the best-fit linear line through the NLCPCA results is about $-0.56 \mathrm{rad} \mathrm{yr}^{-1}$, corresponding to a cycle return period of some 11.5 years, consistent with an earlier CPCA based estimate (Ruessink et al., 2003, 2004).

\subsection{Hasaki}

The best NLCPCA solution for Hasaki was found with $q=6$ and $P=0.01$. The variance explained by the NLCPCA mode 1 was $78.8 \%$, about $6 \%$ more than explained by the leading NLPCA.cir $(q=4$ and $P=0.02)$ and CPCA modes (Table 2). The NLCPCA explaining more variance than CPCA indicates that the underlying signal at Hasaki, just as at Egmond, has considerable non-linear variability. NLCPCA performs very well in the bar zone $(x>\approx 200 \mathrm{~m})$ with typical fractions of explained variance larger than $70 \%$ (Fig. 3b) and, as at Egmond, less well in the bar generation zone $(x \approx 100-200 \mathrm{~m})$. Note that for all three methods the fraction of explained variance reduces to near 0 at $x \approx 100 \mathrm{~m}$ (Fig. 3b), the approximate location of the low-tide level, where the subtidal bar zone changes into the intertidal beach. 

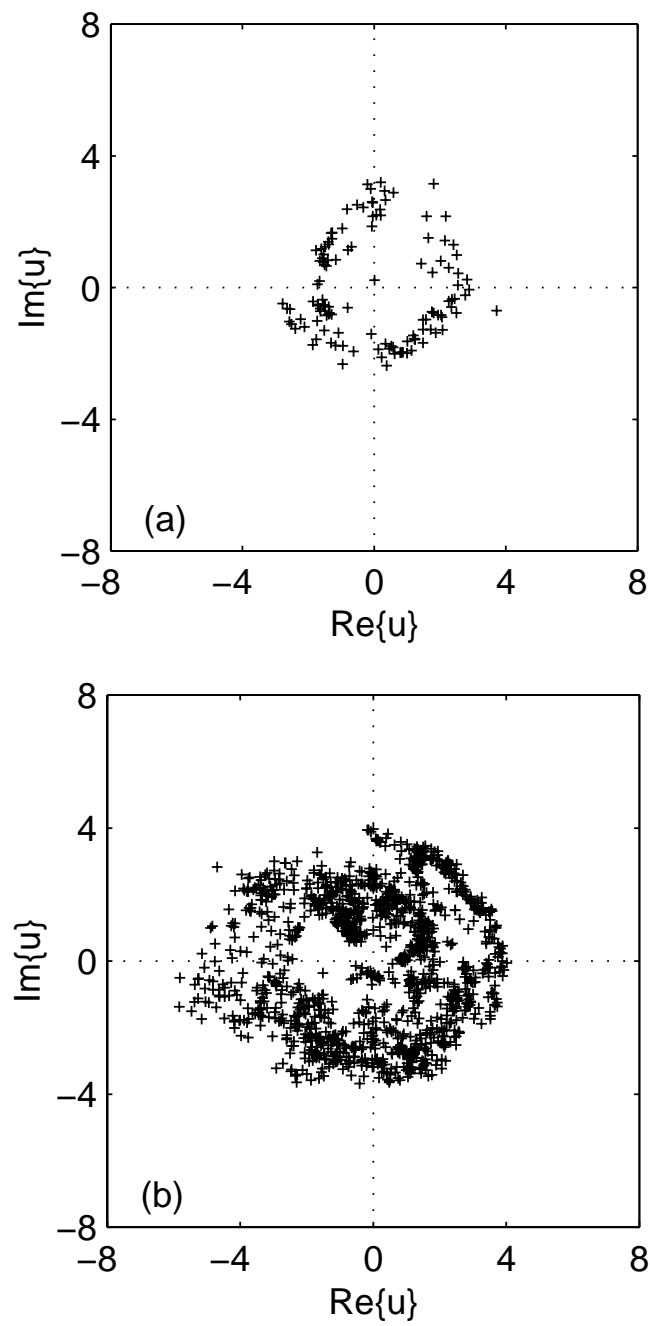

Fig. 7. Imaginary versus real part of NLCPC $u$ at (a) Egmond and (b) Hasaki

Similar to Egmond, the perturbation profiles show remarkable non-linear phase variability. In particular, the inner trough that develops at $x=200 \mathrm{~m}$ once the bar starts moving offshore $(\theta=-0.25 \pi-0.25 \pi$ in Fig. 6$)$ is deeper and broader in the non-linear than in the linear solution, with little differences between the three methods in the shape of the associated sandbar. The deeper trough is also obvious in Fig. 1 by the darker blue colours in the NLCPCA and NLPCA.cir reconstructed data around $x=200 \mathrm{~m}$ in 1987 and 1990 compared to the CPCA solution, in better agreement with the observations. As the bar progresses offshore with a decrease in $\theta$, the NLCPCA and NLPCA.cir reconstructed perturbations become more symmetric, resembling the ones based on the linear mode (Figs. 1 and 6).

Relative to Egmond, the Hasaki bars experiences a somewhat larger amplitude variability (compare Fig. 6a to Fig. 4a), hence the advantage of NLCPCA over NLPCA.cir at Hasaki. The larger variability is also obvious from Fig. 7, which shows scatterplots of the imaginary versus real part of $u$ at Egmond and Hasaki. When successive points at Egmond are connected, the resulting general pattern in Fig. 7a is a clockwise rotating orbit, which represents the interannual offshore propagation of the bars. Temporal changes in the distance $D$ of the points to the origin reflect the temporal amplitude variations. At Egmond, the ratio $R$ of the standard deviation in $D$ to its mean amounts to 0.24 . At Hasaki, the orbit is occasionally interrupted in an anti-clockwise manner, indicative of occasional onshore bar migration, and has a much more jagged structure, caused by larger amplitude variability. At Hasaki, $R$ is larger than at Egmond, about 0.36 .

The amplitude variability at Hasaki appears to be largely linear, however, as the perturbation shapes in each NLCPCA $\theta$ class for the 3 amplitude groups are (besides the magnitudes) about the same (Fig. 6a) and comparable to the corresponding CPCA situation (Fig. 6b). The CPCA and NLPCA.cir explained variances are similar for different reasons: the CPCA explained variance is contributed from the linear amplitude and phase variability, while that of the NLPCA.cir is due to non-linear phase variability only. Thus, the extra information captured by the NLCPCA method (Table 2) is due to temporal amplitude variability missed by the NLPCA.cir method and the non-linear spatial phase variability missed by the CPCA method. As for Egmond, $\theta$ from the 3 linear and non-linear methods are closely alike (not shown, linear correlation coefficients exceed 0.99) and suggest a cycle return period of about 1 year.

\subsection{Duck}

The best NLCPCA solution of Duck was found with $q=4$ and $P=0.01$. The variance explained by this solution was $60.7 \%$ (Table 2), slightly more than the leading linear mode and some $8 \%$ more than the leading NLPCA.cir mode. The relatively close variances explained by the NLCPCA and CPCA, their about equal cross-shore distribution (Fig. 3c), and their very similar perturbation profiles (Figs. 8a and b) indicate the underlying signal at Duck to be essentially linear. The explained variance of NLPCA.cir being lower than in both the CPCA and NLCPCA methods suggests the need to model amplitude variability and the lack of significant non-linearity in the phase variability. At Duck, $\theta$ from the 3 methods are essentially identical and reveal a cycle return period of about 3.5 years.

\section{Discussion and conclusions}

In this paper we have applied NLCPCA to three bathymetric data sets characterized by interannual repetitive offshoredirected sandbar behavior to investigate how effective this method is in comparison to CPCA and NLPCA.cir in representing propagating phenomena. Basically, NLCPCA overcomes deficiencies in both CPCA and NLPCA.cir, in the sense that CPCA can capture phase and amplitude variability only linearly and NLPCA.cir cannot model amplitude variability. Nonetheless, results for the three sites are remarkably 

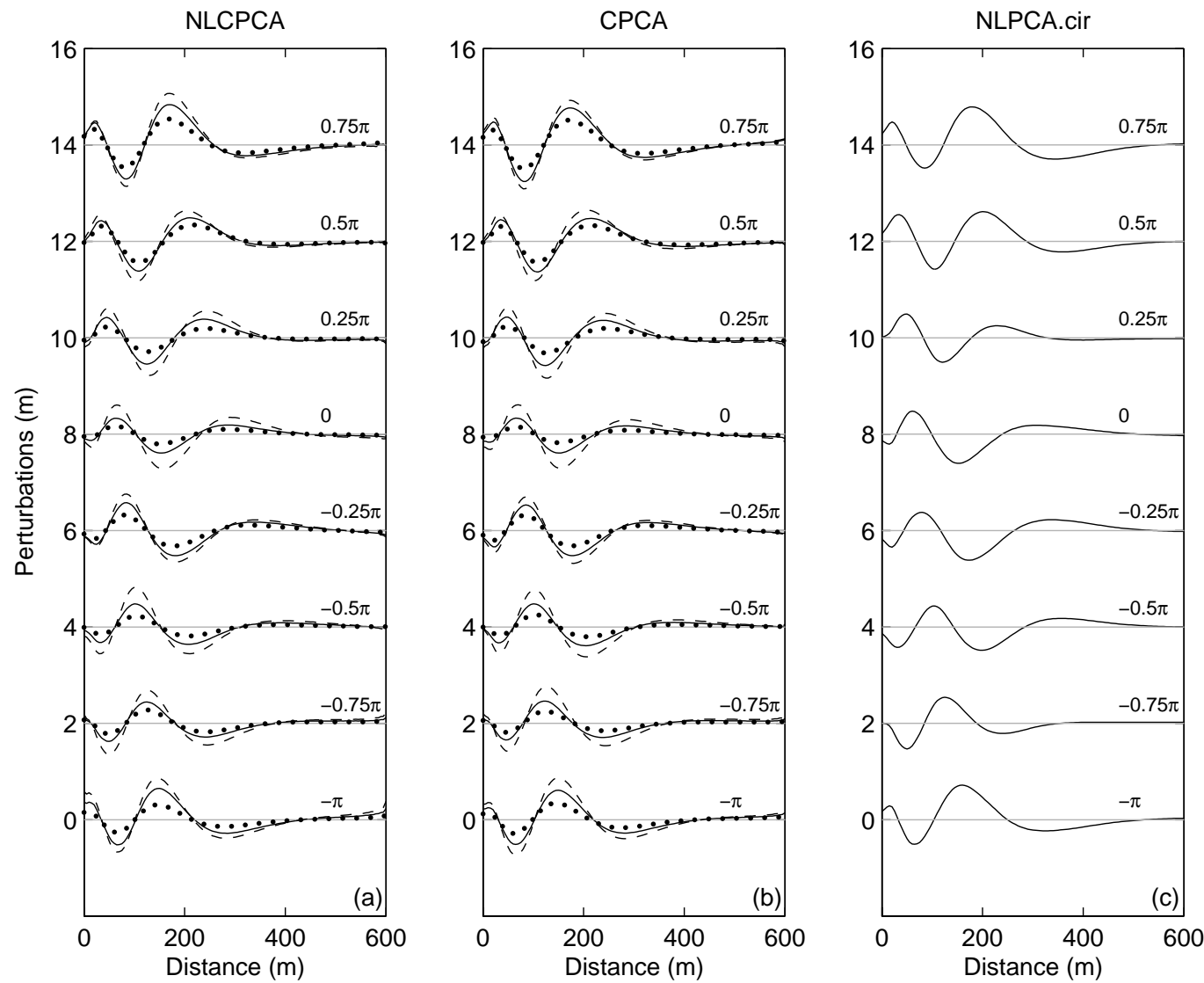

Fig. 8. Sequence of perturbation profiles at Duck in $\pi / 4$-wide $\theta$ classes centered around $\theta=-\pi$ to $\theta=0.75 \pi$ based on (a) NLCPCA mode 1 , (b) CPCA mode 1 and (c) NLPCA.cir mode 1. The three lines in each phase class in (a) and (b) represent the mean perturbation profile for the (dotted line) lower 1/3, (solid line) middle $1 / 3$ and (dashed line) upper $1 / 3$ of the temporal amplitudes in that class. The results for each phase class have been vertically shifted for better visualization.

different. At Egmond and Hasaki the data contains considerable non-linear phase variability, which causes the NLCPCA mode 1 reconstructed data to match the observed data better than CPCA (Fig. 1). At Egmond the offshore propagating bar is higher and steeper than its linear counterpart, and the associated trough is broader and shallower. There is, in addition, a hint of non-linear temporal amplitude variability at Egmond, in particular when the bar is located in the mid-nearshore. At Hasaki the trough that forms near the water line following bar generation is deeper and broader than modelled with CPCA. At Duck non-linearity is found neither in the spatial phase nor in the temporal amplitude variability. Why physically results between the sites differ is not understood.

Only at Hasaki the NLCPCA method explains more variance in a single mode than the other two methods. Here, CPCA does not capture the non-linear spatial phase variability, whereas NLPCA.cir lacks the ability to model temporal amplitude variability. At Egmond, amplitude variability is relatively low, and the NLCPCA performs only marginally better than NLPCA.cir. Finally, at Duck, NLCPCA performs about the same as CPCA as both spatial phase and temporal amplitude variability appear to be linear.
In any propagating phenomena, it is difficult to know the structure of the data in advance as to which one of the three models should be used. In this study the simplest model representing well the data structure at Duck, Egmond and Hasaki is CPCA, NLPCA.cir and NLCPCA, respectively. To avoid choosing a model, the generalized NLCPCA model can be used for all 3 cases.

Acknowledgements. We are indebted to all those involved in the data collection, without whom our present (and earlier) work on sandbars would never have been possible. The Dutch Rijkswaterstaat, Y. Kuriyama (PARI, Japan), and the U.S. Army Corps of Engineers Field Research Facility generously provided the Egmond, Hasaki, and Duck data, respectively. SSPR and WWH were supported by the Natural Sciences and Engineering Research Council of Canada and the Canadian Foundation for Climate and Atmospheric Sciences. BGR was funded by the Netherlands Organisation for Scientific Research under project 864.04.007.

Edited by: B. D. Malamud

Reviewed by: one referee 


\section{References}

Birkemeier, W. A. and Mason, C.: The CRAB: A unique nearshore surveying vehicle, J. Surv. Eng., 110, 1-7, 1984.

Bishop, C. M.: Neural Networks for Pattern Recognition, Oxford University Press, Oxford, 1995.

Gardner, M. W. and Dorling, S. R.: Artificial neural networks (the multilayer perceptron)-a review of applications in the atmospheric sciences, Atmospheric Environment, 32, 2627-2636, 1998.

Hamilton, K. and Hsieh, W. W.: Representation of the QBO in the Tropical stratospheric wind by nonlinear principal component analysis, J. Geophys. Res., 107, doi:10.1029/2001JD001 250, 2002.

Horel, J. D.: Complex principal component analysis: theory and examples, J. Climate Appl. Meteorol., 23, 1660-1673, 1984.

Hsieh, W. W.: Nonlinear principal component analysis by neural networks, Tellus, 53A, 599-615, 2001.

Hsieh, W. W.: Nonlinear multivariate and time series analysis by neural network methods, Rev. Geophys., 42, RG1003, doi:10.1029/2002RG000 112, 2004.

Hsieh, W. W. and Tang, B.: Applying neural network models to prediction and data analysis in meteorology and oceanography, Bulletin of the American Meteorological Society, 79, 1855-1870, 1998.

Jolliffe, I. T.: Principal Component Analysis, Springer Verlag, Berlin, 2002.

Katoh, K. and Yanagishima, S.: Changes of sand grain distribution in the surf zone, in Proc. Coastal Dynamics'95, 639-650, ASCE, New York, 1995.

Kirby, M. J. and Miranda, R.: Circular nodes in neural networks, Neural Comp., 8, 390-402, 1996.

Kramer, M. A.: Nonlinear principal component analysis using autoassociative neural networks, AIChE Journal, 37, 233-243, 1991.

Kuriyama, Y.: Medium-term bar behavior and associated sediment transport at Hasaki, Japan, J. Geophys. Res., 107, doi:10.1029/2001JC000 899, 2002.

Malthouse, E. C.: Limitations of nonlinear PCA as performed with generic neural networks, IEEE Transactions on neural networks, 9, 165-173, 1998.
Plant, N. G., Holman, R. A., Freilich, M. H., and Birkemeier, W. A.: A simple model for interannual sandbar behavior, J. Geophys. Res., 104, 15 755-15 776, 1999.

Rattan, S. S. P. and Hsieh, W. W.: Nonlinear complex principal component analyis of the tropical Pacific interannual wind variability, Geophys. Res. Lett., 31, L21 201, doi:10.1029/2004GL 020 446, 2004.

Rattan, S. S. P. and Hsieh, W. W.: Complex-valued neural networks for nonlinear complex principal component analysis, Neural Networks, 18, 61-69, doi:10.1016/j.neunet.2004.08.002, 2005.

Ruessink, B. G. and Kroon, A.: The behaviour of a multiple bar system in the nearshore zone of Terschelling: 1965-1993, Marine Geology, 121, 187-197, 1994.

Ruessink, B. G., Wijnberg, K. M., Holman, R. A., Kuriyama, Y., and Van Enckevort, I. M. J.: Intersite comparison of interannual nearshore bar behavior, J. Geophys. Res., 108, doi:10.1029/2002JC001 505, 2003.

Ruessink, B. G., Van Enckevort, I. M. J., and Kuriyama, Y.: Nonlinear principal component analysis of nearshore bathymetry, Marine Geology, 203, 185-197, 2004.

Shand, R. D. and Bailey, D. G.: A review of net offshore bar migration with photographic illustrations from Wanganui, New Zealand, J. Coast. Res., 15, 365-378, 1999.

Shand, R. D., Bailey, D. G., and Shephard, M. J.: An inter-site comparison of net offshore bar migration characteristics and environmental conditions, J. Coast. Res., 15, 750-765, 1999.

Strang, G.: Linear Algebra and its Applications, Harcourt, Brace, Jovanovich Publishers, San Diego, 1988.

Thornton, E. B. and Guza, R. T.: Energy saturation and phase speeds measured on a natural beach, J. Geophys. Res., 87, 94999508, 1982.

Von Storch, H. and Zwiers, F. W.: Statistical analysis in climate research, Cambridge University Press, Cambridge, 1999.

Wijnberg, K. M. and Terwindt, J. H. J.: Extracting decadal morphological behaviour from high-resolution, long-term bathymetric surveys along the Holland coast using eigenfunction analysis, 126, 301-330, 1995. 